\title{
Author Correction: Saraswati the wide-reaching
}

Marios Karouzos

Correction to: Nature Astronomy 1, 564 (2017); published online 21st August 2017.

In the version of this Research Highlight originally published, in the second paragraph the number of galaxy clusters in the Saraswati supercluster was incorrectly stated. The sentence has been updated to read: "This superstructure contains more than 40 confirmed galaxy clusters and is surrounded by vast galactic voids." This has been corrected in all versions of the Research Highlight.

Published online: 25 October 2017

https://doi.org/10.1038/s41550-017-0300-4 